\title{
Ionised Accretion Discs in Active Galactic Nuclei: The Effects of a Lamppost with a Variable Height
}

\author{
D. R. Ballantyne \\ Center for Relativistic Astrophysics, School of Physics, Georgia Institute of Technology, 837 State Street, Atlanta, GA 30332-0430, USA
}

Accepted XXX. Received YYY; in original form ZZZ

\begin{abstract}
The X-ray emitting corona irradiates and ionises the surface of the inner accretion disc in Active Galactic Nuclei (AGNs). The ionisation parameter of the inner disc at a radius $r$ from the black hole, $\xi(r)$, can be used to infer information about the location of the corona. Here, a new formula is derived that predicts $\xi(r, h)$ for a disc irradiated by a X-ray source at a height $h$ above the black hole symmetry axis (i.e., a lamppost geometry). The equation is independent of the black hole mass and the X-ray spectrum, and accounts for the effects of gravitational light-bending on the ionisation state and a variable coronal dissipation factor. We predict a strong ionisation gradient across the surface of the inner disc that depends on the black hole spin and lamppost height. For a fixed $h$, the ionisation parameter is also expected to increase as $\lambda^{3}$, where $\lambda$ is the observed bolometric Eddington ratio of the AGN. Comparing this formula to the observed $\xi-\lambda$ relationship for Mrk 335 yields $h \propto \lambda^{0.5-0.6}$, supporting the view of a dynamic X-ray corona in AGNs.
\end{abstract}

Key words: galaxies: active $-\mathrm{X}$-rays: galaxies - accretion, accretion discs - galaxies: Seyfert - galaxies: individual (Mrk 335) - relativistic processes

\section{INTRODUCTION}

X-ray spectroscopy is an invaluable probe of the physics of accreting supermassive black holes in active galactic nuclei (AGNs). The X-ray spectra of AGNs are largely comprised of two components: a cutoff power-law and a reflection spectrum that is produced by the reprocessing of the power-law in dense matter (e.g., Fabian \& Ross 2010). The observed relativistically broadened $\mathrm{Fe} \mathrm{K} \alpha$ lines indicate that the inner regions of the accretion disc are responsible for at least some of this reprocessing. Thus, as supported by its rapid variability (e.g., Kara et al. 2016), the illuminating power-law source must also arise from small radii, close to the central black hole. The power-law is consistent with arising from the Compton up-scattering of ultraviolet photons from the accretion disc in a tenuous and hot electron corona (e.g., Haardt \& Maraschi 1991, 1993). Therefore, the reflection signal and coronal emission are deeply intertwined within the total spectrum, and one of the challenges of X-ray observations of AGNs is to extract the physical conditions of the disc-corona system from the data.

One property of the accretion disc that can yield important insights is the ionisation state produced by the irradiating power-law. The surface ionisation state is sensitive to both the disc structure and the coronal illumination - most simply described by an ionisation parameter, $\xi=4 \pi F_{X} / n_{\mathrm{H}}$, where $F_{X}$ is the X-ray flux incident on gas with hydrogen number density $n_{\mathrm{H}}$. Calculations of X-ray reprocess-

^ E-mail: david.ballantyne@physics.gatech.edu ing from the surface of accretion discs show that changes in $\xi$ can have a significant and measurable effect on the observed reflection spectrum (e.g., García et al. 2013, 2014). Therefore, measurements of $\xi$ from AGNs have the potential to probe the illumination and structure of the disc-corona system close to the black hole.

Interpretation of any measured values of $\xi$ are hampered by uncertainties in the location and geometry of the corona, as well as the exact physical structure of accretion discs at distances $r \lesssim 10 r_{g}$ from the black hole $\left(r_{g}=G M / c^{2}\right.$ is the gravitational radius of a black hole of mass $M$ ). As a first step, Ballantyne, McDuffie \& Rusin (2011) used a Shakura \& Sunyaev (1973) based $\alpha$-disc model to derive an analytical estimate of how $\xi(r)$ should depend on various interesting physical parameters such as black hole spin $(a)$, coronal dissipation fraction $(f$; Svennson $\&$ Zdziarski 1994), and Eddington ratio $\left(\lambda=L_{\text {bol }} / L_{\text {Edd }}\right.$, where $L_{\mathrm{bol}}$ is the bolometric luminosity and $L_{\mathrm{Edd}}=4 \pi G M m_{p} c / \sigma_{\mathrm{T}}$ is the Eddington luminosity). The calculation predicted a strong correlation between $\xi(r)$ and $\lambda$ which was confirmed using a selection of literature values, although the data indicated a flatter slope than the prediction.

The derivation by Ballantyne et al. (2011) assumed a compact, geometrically thick corona (where the coronal scale-height $h=r$ ) and neglected relativistic effects that could enhance the irradiating flux. In recent years, $\mathrm{Fe} \mathrm{K} \alpha$ reverberation measurements and quasar micro-lensing studies point to a coronal geometry that is very compact ( $r \lesssim 10 r_{g}$ ), and may be better approximated by a lamppost geometry where the $\mathrm{X}$-ray source is situated above the spin 
axis of the black hole (Reis \& Miller 2013; Kara et al. 2016). Here, we re-derive the expected $\xi(r)$ relationship for a radiation-pressure dominated accretion disc irradiated by a lamppost X-ray source, and include the effects of relativistic light-bending. As is shown below, with the appropriate datasets the predicted $\xi(r, h)$ relationship can be used to determine the potential evolution of the X-ray emitting corona. The next section describes the derivation of the new $\xi(r, h)$ relationship, with the results analyzed in Sect. 3, including an application to the AGN Mrk 335. A summary and conclusions are presented in Sect. 4.

\section{CALCULATIONS}

As in Ballantyne et al. (2011) we begin by writing $\xi=4 \pi m_{p} \rho^{-1} F_{X}$, where $m_{p}$ is the mass of a proton and $\rho$ is the density of a radiation-pressure dominated $\alpha$ disc (Shakura \& Sunyaev 1973), corrected for a non-zero coronal dissipation fraction ${ }^{1}$ by following Svennson \& Zdziarski (1994):

$$
\begin{aligned}
\rho= & \left(2.23 \times 10^{-6}\right)\left(\frac{\eta}{0.1}\right)^{2}\left(\frac{\alpha}{0.1}\right)^{-1}\left(\frac{M}{M_{\odot}}\right)^{-1} \lambda^{-2}\left(\frac{r}{r_{g}}\right)^{3 / 2} \\
& \times R_{Z}^{2} R_{T} R_{R}^{-3}(1-f)^{-3} \mathrm{~g} \mathrm{~cm}^{-3} .
\end{aligned}
$$

In this equation, $\eta$ is the radiative efficiency of the accretion process, and $\left(R_{R}, R_{z}, R_{T}\right)$ are relativistic corrections to the Newtonian $\alpha$-disc equations that depend on $a$ and $r$ (Krolik 1999). We assume this is the density responsible for X-ray reflection at the disc surface.

The problem is now to determine the total X-ray flux $F_{X}$ incident on the surface of the disc at a radius $r$ from a X-ray source radiating at a height $h$ (in units of $r_{g}$ ) above the black hole while including the relativistic focusing effects. Following Vincent et al. (2016), we define a lamppost X-ray source emitting isotropically in its rest-frame with a spectrum $v_{\mathrm{src}}^{-\beta}$. The flux normal to the disc surface at some radius $r$ is then

$F_{X, v_{\text {disc }}}(r)=A v_{\text {disc }}^{-\beta} \mathcal{F}(r, h)$,

where $A$ is a normalization constant, and $\mathcal{F}(r, h)$ is a function that describes the illumination profile of the disc irradiated by a lamppost source and includes the effects of light-bending. The measured frequency of a photon striking the disc, $v_{\text {disc }}$ is related to its frequency at the source $v_{\text {src }}$ in a lamppost geometry by (Dauser et al. 2013)

$g_{\mathrm{lp}}=\frac{v_{\mathrm{disc}}}{v_{\mathrm{src}}}=\frac{r^{3 / 2}+a}{\sqrt{r^{3}+2 a r^{3 / 2}-3 r^{2}}} \sqrt{\frac{h^{2}+a^{2}-2 h}{h^{2}+a^{2}}}$.

The function $\mathcal{F}(r, h)$ has been computed using ray-tracing simulations by several groups (e.g., Fukumura \& Kazanas 2007; Chainakun \& Young 2012; Wilkins \& Fabian 2012; Dauser et al. 2013; Vincent et al. 2016). Writing $\mathcal{F}(r, h)=F(r, h) g_{1 \mathrm{p}}^{1+\beta}$, Fukumura \& Kazanas (2007) provide fitting formulas for the shape of the irradiation pattern $F(r, h)$ for $3 \leqslant\left(h / r_{g}\right) \leqslant 100$ and $r>1.15 r_{g}$ (their Eqs. 10 and 11). With this identification, Eq. 2 can be integrated over frequency to yield the total X-ray flux normally incident on the disc at radius $r$ :

$F_{X}=A F(r, h) g_{\mathrm{lp}}^{1+\beta}\left(v_{\text {disc, } \max }^{1-\beta}-v_{\text {disc, } \min }^{1-\beta}\right)(1-\beta)^{-1}$.

1 This is a fixed fraction of the total dissipation profile, which is centrally concentrated (Shakura \& Sunyaev 1973), and is a decent approximation to a lamppost corona.
To set the normalization $A$, we make use of the X-ray luminosity of the lamppost as seen by the accretion disc, and can be defined by integrating the flux (Eq. 4) over the entire accretion disc, and converting the frequencies to their values in the source frame (using Eq. 3):

$L_{X}=A\left(v_{\text {src, } \max }^{1-\beta}-v_{\text {src, } \min }^{1-\beta}\right)(1-\beta)^{-1} \mathcal{A}$,

where

$\mathcal{A}=\int_{r_{\text {in }}}^{r_{\text {out }}} F(r, h) g_{\text {lp }}^{2} d S(r)$

and

$d S(r)=2 \pi r \sqrt{\frac{r^{2}+a^{2}+2 a^{2} / r}{r^{2}-2 r+a^{2}}} d r$

is the proper element of disc area at the midplane (Vincent et al. 2016). Equations 4 and 5 can be used to eliminate $A$ and obtain a final expression for the $\mathrm{X}$-ray flux incident on the disc at radius $r$,

$F_{X}(r)=\frac{L_{X} F(r, h) g_{\mathrm{lp}}^{2}}{z(M) \mathcal{A}}$,

where

$z(M)=\left(\frac{G M_{\odot}}{c^{2}}\right)^{2}\left(\frac{M}{M_{\odot}}\right)^{2}$

is a factor that converts the area to physical units (e.g., $\mathrm{cm}^{2}$ ).

As mentioned above, $L_{X}$ is the total X-ray luminosity seen by the disc, and will be directly related to the accretion power dissipated in the corona, $L_{X}=f L_{\text {bol }}$. With this identification, the expression for the flux on the disc surface (Eq. 8) can be combined with the one for the disc density (Eq. 1), to yield the desired equation for the disc ionisation parameter at radius $r$ when illuminated by a lamppost corona at height $h$ while including the effects of gravitational lightbending:

$$
\begin{aligned}
\xi(r, h)= & \left(5.44 \times 10^{10}\right)\left(\frac{\eta}{0.1}\right)^{-2}\left(\frac{\alpha}{0.1}\right) \lambda^{3}\left(\frac{r}{r_{g}}\right)^{-3 / 2} R_{z}^{-2} R_{T}^{-1} \\
& \times R_{R}^{3} f(1-f)^{3} F(r, h) g_{l p}^{2} \mathcal{A}^{-1} \mathrm{erg} \mathrm{s} \mathrm{cm}^{-1} .
\end{aligned}
$$

Note that $\xi$ only depends on the black hole spin and not on its mass or the spectral shape of the illuminating X-rays. The equation is valid for $3 r_{g} \leqslant h \leqslant 100 r_{g}$ and $r>1.15 r_{g}$, assuming $a$ is large enough to ensure the disc extends to such small radii. In what follows, $\mathcal{A}$ is calculated with $r_{\text {out }}=200 r_{g}$ and $r_{\text {in }}$ is set to the innermost stable circular orbit (ISCO).

\section{RESULTS}

Figure 1 plots $\xi(r, h)$ across the inner accretion disc predicted by Eq. 10 for lampposts with 4 different heights. For the purpose of this example, a fixed coronal dissipation fraction of $f=0.45$ (Vasudevan \& Fabian 2007) and Eddington ratio $\lambda=0.1$ are used for the calculations. Unlike earlier calculations in the literature (e.g., Svoboda et al. 2012; Chainakun \& Young 2012, 2015), these ionisation profiles include the effects of both relativistic light-bending and the density profile of an accretion disc model. The combination of the radial dependicies of both the disc illumination and density leads to the predicted shape of the ionisation profiles.

Apart from the one shown using the dotted line, each of the ionisation profiles shown in Fig. 1 exhibits a break at $r=4 r_{g}$. The break results from setting the $\left(R_{z}, R_{T}, R_{R}\right)$ factors at $r<4 r_{g}$ to their values 


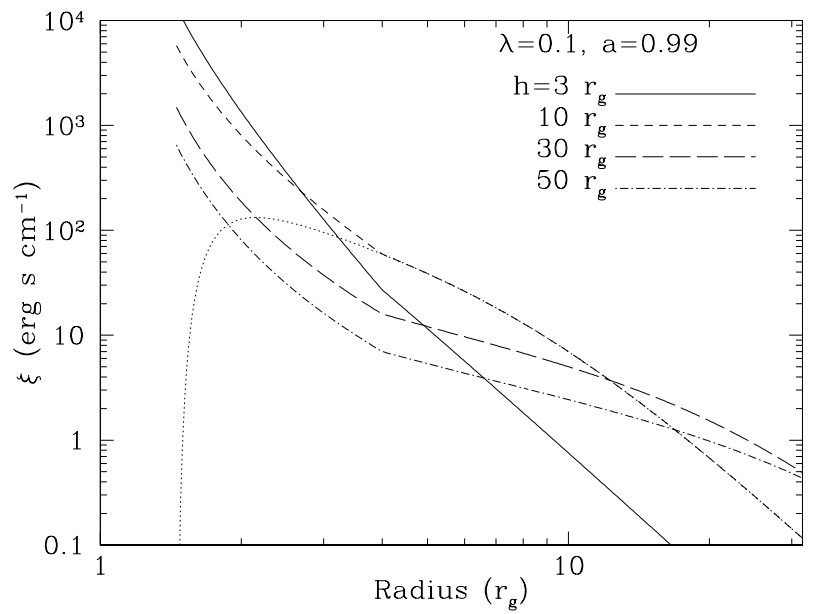

Figure 1. The predicted $\xi(r)$ of an irradiated accretion disc from a lamppost X-ray source situated at different heights above the black hole (Eq. 10). For this illustration, $\lambda=0.1, a=0.99, f=0.45$ (Vasudevan \& Fabian 2007), $\alpha=0.1$ and $\eta=0.1$. The different line styles indicate the various source heights considered. The $\left(R_{z}, R_{T}, R_{R}\right)$ factors are fixed at their $r=4 r_{g}$ values for $r<4 r_{g}$ to avoid an unphysical turnover in $\xi$. The dotted line shows an example of this behavior for $h=10 r_{g}$.

at $r=4 r_{g}$ to correct for the divergence of $R_{R}$ and $R_{T}$ (Krolik 1999) as the radius approaches the ISCO. As $r$ decreases past $4 r_{g}, R_{R}$ and $R_{T}$ plunge toward zero, causing the density to become infinitely large (Eq. 1) and hence $\xi(r, h)$ approaches zero at the inner edge of the disc (dotted line in Fig. 1; see also Keek \& Ballantyne 2016, Fig. 6). However, as shown by numerical simulations of accretion flows, this behaviour is unphysical as the disc density actually drops as the gas passes through the ISCO (e.g., Morales Teixeira et al. 2014), and the ionisation parameter should therefore increase at small radii (Reynolds \& Fabian 2008). After fixing the factors, the predicted ionisation parameters increases strongly to small radii, and more closely follows the increase in illumination predicted by light-bending models (e.g., Dauser et al. 2013); however, there is still some effect from the radial dependence of the density. Thus, our correction to the relativistic factors should give a more accurate portrayal of $\xi(r)$ close to the ISCO. Ultimately, numerical simulations of the ionisation profile along the disc surface are needed to precisely determine $\xi(r)$.

Figure 1 clearly shows the impact of gravitational lightbending on the ionisation state of the inner disc. For a source height of $3 r_{g}$, most of the released luminosity of the lamppost is focused onto the disc close to the ISCO, with only a small amount of flux irradiating the disc beyond $\sim 10 r_{g}$. As the lamppost is raised higher, the amount of flux reaching larger radii increases, giving rise to the seemingly paradoxical condition where the source appears brighter to the disc even though it is moving further away (Figure 2).

A significant ionisation gradient on the surface of the inner accretion disc is predicted, with a slope that depends strongly on the height of the lamppost. This gradient is a combination of both the illumination pattern along the disc (often called the disc emissivity when computing the relativistic effects on the reflected spectrum; Dauser et al. 2013), and the radial density structure of the disc. To quantify the gradient in the models shown in Fig. 1, the slope $b$ of the relation $\log \xi \propto b \log \left(r / r_{g}\right)$ was computed over $2 \leqslant\left(r / r_{g}\right) \leqslant 20$ as a function of source height. Figure 3 shows the resulting ionisation gradient varies from $\xi \propto\left(r / r_{g}\right)^{-4.3}$ for $h=3 r_{g}$ to $\xi \propto\left(r / r_{g}\right)^{-1.6}$

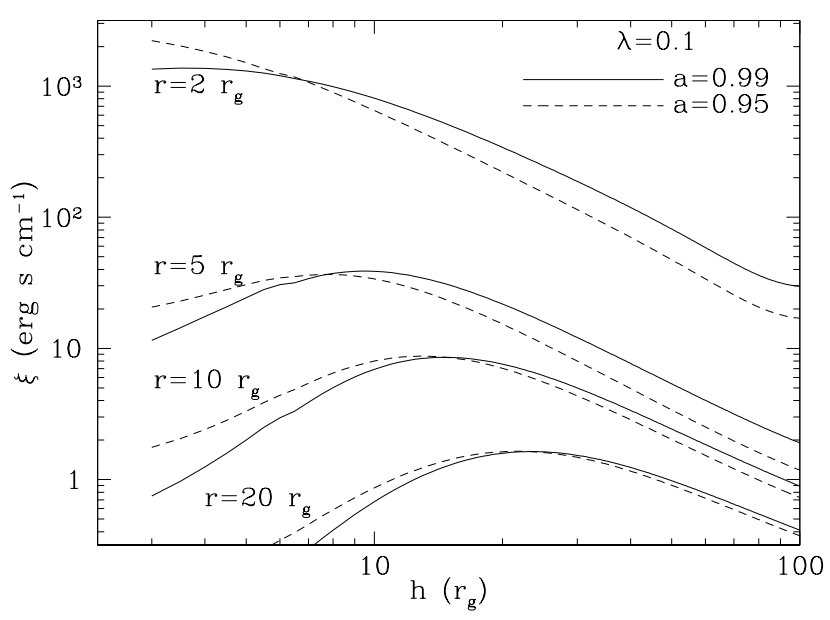

Figure 2. The variation of $\xi$ with lamppost height $h$ at four radii along the accretion disc. The model parameters are the same as described in Fig. 1. The ionisation parameter close to the ISCO falls as the source height increases. However, because of the reduction of gravitational focusing to small radii, $\xi(r, h)$ at $r \gtrsim 5 r_{g}$ actually rises as $h$ increases before reaching a maximum and then dropping. The dashed lines show results for a model with a slightly lower black hole spin $(a=0.95)$ where the larger ISCO means that more flux can be distributed over the disc leading to slightly elevated values of $\xi$ for low $h$ compared to the high spin model.

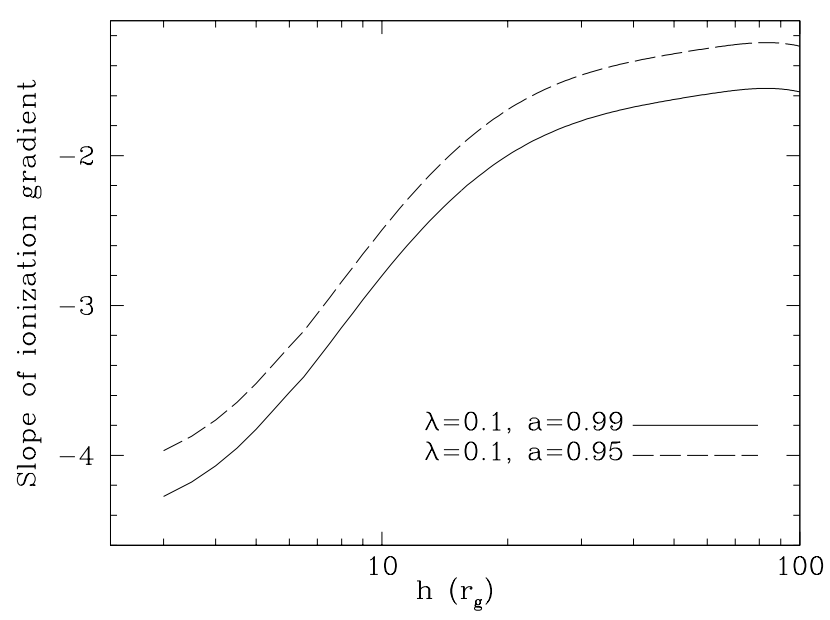

Figure 3. The slope of the disc surface ionisation gradient, defined as $\log \xi \propto b \log \left(r / r_{g}\right)$, predicted by Eq. 10 as a function of the lamppost height. The slope, $b$, is computed between $r=2$ and $20 r_{g}$ for two different black hole spins. The slope of the ionisation gradient increase with the spin because the smaller ISCO allows more radiation to be focused to the center of the disc. Values for $\alpha, \eta$ and $f$ are fixed to $0.1,0.1$, and 0.45 , respectively.

for $h \gtrsim 50 r_{g}$. This result may assist the interpretation of ionisation parameters determined from reflection models fit to AGN X-ray data (e.g., Brenneman \& Reynolds 2009), since the measured value of $\xi$ is actually an average over an unknown ionisation gradient. As the disc emissivity is included in the calculations, Fig. 3 shows that for a lamppost source the observed $\xi$ will be dominated by the ionisation state of the inner accretion disc.

The principle impact of a different black hole spin on $\xi(r, h)$ is the change in the location of the ISCO. This affects how the flux can be distributed along the disc (e.g., Fukumura \& Kazanas 2007) 


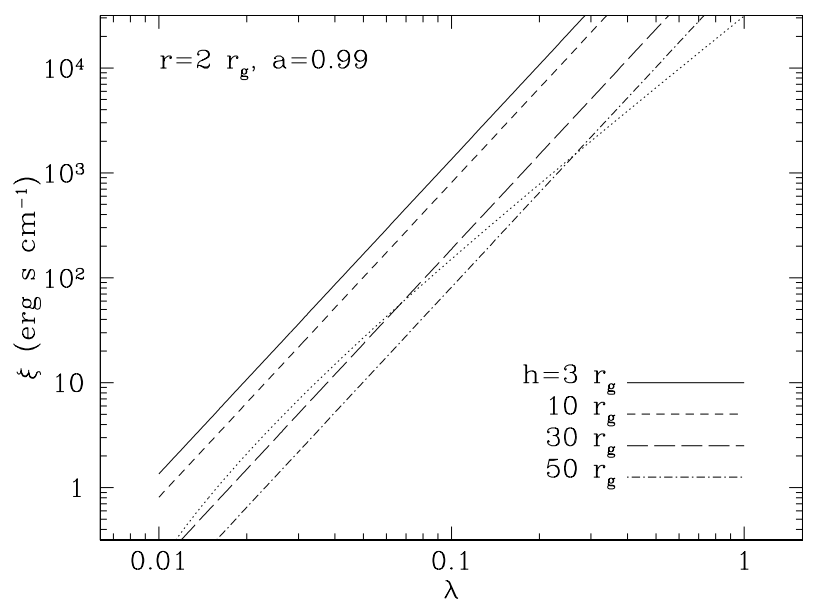

Figure 4. The predicted $\xi-\lambda$ relationship at $r=2 r_{g}$ for different lamppost heights (Eq. 10). All lines, except for the dotted line, plot models where $f=0.45, \eta=0.1$ and $\alpha=0.1$. The dotted line shows how the $h=30 r_{g}$ model changes when $\log f \propto-0.77 \log \lambda($ Stella \& Rosner 1984).

and is illustrated in Figs. 2 and 3. In these plots, the results for $a=0.95\left(r_{\mathrm{ISCO}}=1.94 r_{g}\right)$ and $a=0.99\left(r_{\mathrm{ISCO}}=1.45 r_{g}\right)$ are shown. The disc extends closer to the black hole in the $a=0.99$ case and therefore gravitational light-bending can strongly focus flux to small radii leading to a large $\xi$ at the ISCO and a steep gradient. Therefore, when comparing $\xi$ at the same radius, the lower spin model has the larger $\xi$ when $h$ is small because more flux impacts these radii. When $h$ is larger, the situation reverses, as the high spin model allows for more photon trajectories to impact the inner disc.

Finally, Figure 4 shows the strong dependence that $\xi(r, h)$ is predicted to have on $\lambda$. Equation 10 predicts that $\xi \propto \lambda^{3}$, which is the same dependence as the Ballantyne et al. (2011) relationship. As shown in that paper, this relationship can be altered if some of the parameters of Eq. 10 also depend on $\lambda$. For example, the coronal dissipation is likely a function of the Eddington ratio, and the dotted line in Fig. 4 shows how the predicted $\xi-\lambda$ relationship changes if $\log f \propto-0.77 \log \lambda$ (Stella \& Rosner 1984). Of course, this new prediction of $\xi$ depends significantly on lamppost height $(h)$. Therefore, if the height of the X-ray source changes with $\lambda$ (Kara et al. 2016), then the predicted $\xi-\lambda$ relationship will be affected. Finally, Fig. 4 shows that the predicted ionisation parameter of the inner disc can be very low for Eddington ratios of a few percent - typical values for Seyfert galaxies. Indeed, the model naturally explains why most relativistic lines from Seyfert galaxies arise from neutral iron (e.g., Brenneman \& Reynolds 2009) and why rapidly accreting AGNs, such as Mrk 335 (discussed below) or Ark 564 (Kara et al. 2017) exhibit lines from ionised iron (see also the stacking results of Liu et al. 2016).

\subsection{Application to Mrk 335}

As a specific example of how Eq. 10 can be used to elucidate details of AGN coronae, we consider the case of Mrk 335. This AGN was the subject of a recent study by Keek \& Ballantyne (2016) that selfconsistently modeled a dozen separate X-ray observations with relativistic ionised reflection models. The observations spanned nearly a decade in flux and were collected over a period of 14 years. Thus, they provided many individual snapshots of the state of the inner accretion disc when the source was accreting at different rates. The

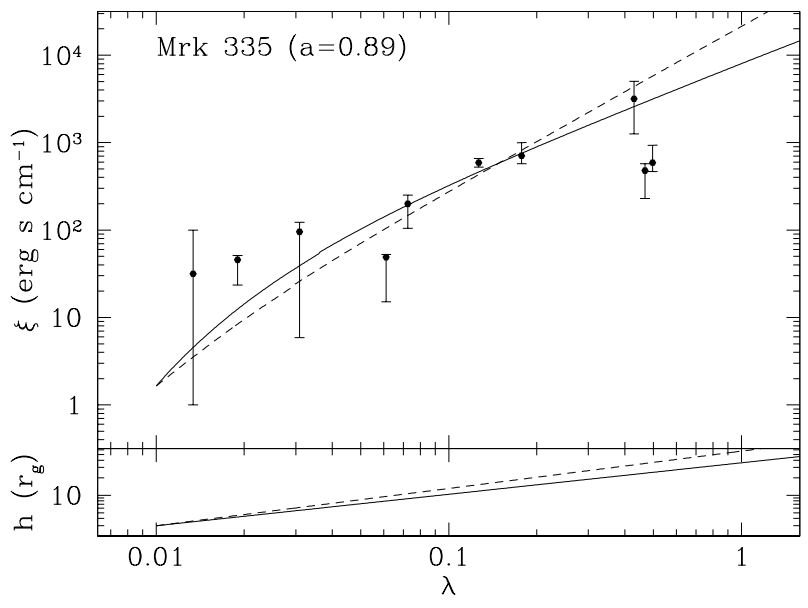

Figure 5. The top panel plots the $\xi$ versus $\lambda$ data derived from spectral fitting of multiple observations of the AGN Mrk 335 (Keek \& Ballantyne 2016). The two lines are models fit to these data using Eq. 10 under the assumption $\log h \propto \beta \log \lambda$. The solid line plots a model where $\log f \propto-0.77 \log \lambda$ (Stella $\&$ Rosner 1984) and the dashed line assumes $f=0.45$ (Vasudevan \& Fabian 2007). Both models are calculated assuming the observed $\xi$ is from the ISCO $\left(r \approx 2.4 r_{g}\right)$ for a black hole with spin $a=0.89$ (Keek \& Ballantyne 2016). In addition, $\eta=0.1, \alpha=0.3$, and $h=3 r_{g}$ for $\lambda=0.01$. The lower panel shows how $h$ varies with $\lambda$ for the two models: $h \propto \lambda^{0.54}$ (solid line) and $h \propto \lambda^{0.64}$ (dashed line).

analysis of Keek \& Ballantyne (2016) found a strong correlation between the measured $\xi$ and the observed flux which, after estimating bolometric corrections, gave a correlation with $\lambda$.

The upper panel of Figure 5 shows the measured Mrk $335 \xi$ $\lambda$ correlation from Keek \& Ballantyne (2016). Overplotted in this panel are two models calculated directly from Eq. 10 and are described in detail below. The comparison between the data and the models is not entirely self-consistent. The quality of the Mrk 335 spectra meant that the disc emissivity needed to be fixed at -3 during spectral fitting. The values of $\xi$ computed from Eq. 10 incorporate the correct emissivity for the lamppost model, which will vary with the height of the X-ray source (Dauser et al. 2013). Because of this inconsistency, the application to Mrk 335 presented here should be considered an illustration of the potential of this analysis for future datasets where the emissivity can be satisfactorily constrained.

To calculate the expected $\xi-\lambda$ relations, $a$ was set to 0.89 , the value determined by the spectral fitting of Keek \& Ballantyne (2016). The measured values of $\xi$ were derived by Keek \& Ballantyne (2016) assuming reflection down to the ISCO with a fixed emissivity of -3 . Therefore, the measured values of $\xi$ are determined from a centrally concentrated spectral model, similar to a lamppost geometry. Figure 3 shows that the ionisation gradient is expected to be steep, and the predicted ionisation parameter from the ISCO should dominate the reflected emission for this geometry. To compare against the data, the two $\xi$ - $\lambda$ models shown in Fig. 5 are therefore computed at the ISCO of an accretion disc around a black hole with spin $a=0.89$ (i.e., $r=2.4 r_{g}$; both models also assume $\eta=0.1)$. The dashed line assumes a constant $f=0.45$ (Vasudevan \& Fabian 2007) and the solid line shows a model where $\log f \propto-0.77 \log \lambda$ (Stella $\&$ Rosner 1984). However, in order to account for the slope of the observed relation ( $\log \xi \propto 1.1 \log \lambda$; Keek \& Ballantyne 2016), the height of the lamppost must also vary with Eddington ratio.

To determine the variation of $h$ with $\lambda$ we assume $\log h \propto$ 
$\beta \log \lambda$. To normalize the relation, $h$ is set to $3 r_{g}$ (the lowest acceptable value for Eq. 10) at $\lambda=0.01$. Even with the X-ray source at such a low height, the predicted $\xi$ at $\lambda \approx 0.01$ is well below the observed data, so $\alpha$ was set to 0.3 , consistent with values found close to the ISCO in numerical simulations of accretion flows (Penna et al. 2013). Chi-square fitting was then performed to find the best $\beta$ for each of the two models. For the model with $f=0.45$ (dashed line), $h \propto \lambda^{0.64}$, while for the model with $\log f \propto-0.77 \log \lambda$ (solid line), $h \propto \lambda^{0.54}$. In both cases, the lower panel of Fig. 5 shows how the height of the lamppost depends on the $\lambda$, reaching heights of $h \sim 30 r_{g}$ when $\lambda \sim 1$. Lamppost heights $\lesssim 30 r_{g}$ are consistent with the coronal distances inferred from X-ray reverberation (Kara et al. 2016; King, Lohfink \& Kara 2017), especially after accounting for the effects of dilution (e.g., Cackett et al. 2014; Chainakun \& Young 2015) that can increase the measured distances by a factor of a few.

An increasing X-ray source height with $\lambda$ in Mrk 335 is consistent with other relationships uncovered in the Keek \& Ballantyne (2016) spectral analysis, such as a smaller reflection fraction at larger $\lambda$. In particular, by using the measured properties of the $\mathrm{X}$ ray power-law, Keek \& Ballantyne (2016) estimated that the coronal temperature was much higher, and the optical depth lower, at large $\lambda$. Combining all these relationships together supports a corona that was compact and relatively cool at low $\lambda$, but is hotter and more extended at high Eddington ratios. The above analysis of the $\xi-\lambda$ plane supports this picture, which is also in broad agreement with the results of Wilkins \& Fabian (2015), who modeled the emissivity profile of the $\mathrm{Fe} \mathrm{K} \alpha$ line to infer a corona in Mrk 335 that evolved in both the vertical and radial directions.

\section{SUMMARY AND CONCLUSIONS}

In this work, a formula for the ionisation parameter of the surface of an irradiated AGN accretion disc, $\xi(r, h)$, has been derived for a X-ray source placed at a height $h$ above the symmetry axis of the black hole (Eq. 10). This equation takes into account the effects of gravitational light-bending and focusing of radiation onto the disc (e.g., Vincent et al. 2016), and is independent of black hole mass and the spectrum produced by the X-ray source. Therefore, it can be used to test models of AGN coronae within a lamppost geometry.

As shown in Figs. 1-3, the theory predicts a strong ionisation gradient across the disc surface that depends on $h$ and the spin of the black hole, $a$. A more rapidly spinning black hole has a smaller ISCO, and therefore ionised reflection is enhanced close to the ISCO by gravitational focusing leading to a steeper ionisation gradient for larger values of $a$. As the height of the X-ray source increases, the reduction of light-bending at small radii first causes $\xi$ to grow at small radii before eventually dropping with even larger $h$ (Fig. 2).

The $\xi(r, h)$ equation derived here predicts a strong dependence on $\lambda$ (Fig. 4). However, this dependence can be modified if the height of the lamppost varies with $h$. This was illustrated by comparing the predictions of Eq. 10 with the $\xi-\lambda$ data determined for the AGN Mrk 335 (Keek \& Ballantyne 2016). Figure 5 showed that if $h \propto \lambda^{0.5-0.6}$ the predicted $\xi-\lambda$ relation could broadly follow the observed datapoints. As future X-ray spectroscopic studies of AGNs become more precise, comparisons between how $\xi$ varies with $\lambda$ with the theory presented here could provide constraints on the evolution of the X-ray source in the lamppost model.

The conclusion from the Mrk 335 comparison is that the height of the corona, at least in the lamppost model, will increase with Eddington ratio or AGN flux state. This result agrees with the recent work of King et al. (2017) who found that the corona height increased with the AGN radio jet power, which may indicate a connection between the base of the jet and the X-ray source (e.g., Markoff \& Nowak 2004). Enhanced radiation pressure from the disc may also cause the corona to be at a larger heights during higher accretion rates (Beloborodov 1999). Additional relations based on the measured reflection fraction also indicate a more distant X-ray source at larger $\lambda$ (Keek \& Ballantyne 2016; King et al. 2017). Future work will focus on extending the theory presented here to pre$\operatorname{dict} \xi(r, h)$ for a moving X-ray source, and to investigate calculating a simultaneous prediction of the reflection fraction.

\section{REFERENCES}

Ballantyne D.R., McDuffie J.R., Rusin J.S., 2011, ApJ, 734, 112

Beloborodov A.M., 1999, ApJ, 510, L123

Brenneman L.W., Reynolds C.S., 2009, ApJ, 702, 1367

Cackett E.M., Zoghbi A., Reynolds C., Fabian A.C., Kara E., Uttley P., Wilkins D.R., 2014, MNRAS, 438, 2980

Chainakun P., Young A.J., 2012, MNRAS, 420, 1145

Chainakun P., Young A.J., 2015, MNRAS, 452, 333

Dauser T., Garcia J., Wilms J., Böck M., Brenneman L.W., Falanga M., Fukumura K., Reynolds C.S., 2013, MNRAS, 430, 1694

Fabian A.C., Ross R.R., 2010, Space Sci. Rev., 157, 167

Fukumura K., Kazanas D., 2007, ApJ, 664, 14

García J., Dauser T., Reynolds C.S., Kallman T.R., McClintock J.E., Wilms J., Eikmann W., 2013, ApJ, 768, 146

García J. et al., 2014, ApJ, 782, 76

Haardt F., Maraschi L., 1991, ApJ, 380, L51

Haardt F., Maraschi L., 1993, ApJ, 413, 507

Haardt F., Maraschi L., Ghisellini G., 1994, ApJ, 432, L95

Kara E., Alston W.N., Fabian A.C., Cackett E.M., Uttley P., Reynolds C.S., Zoghbi A., 2016, MNRAS, 462, 511

Kara E., Garcia J.A., Lohfink A., Fabian A.C., Reynolds C.S., Tombesi F., Wilkins D.R., 2017, MNRAS, 468, 3489

Keek L., Ballantyne D.R., 2016, MNRAS, 456, 2722

King A.L., Lohfink A., Kara E., 2017, ApJ, 835, 226

Krolik, J.H., 1999, Active Galactic Nuclei: From the Central Black Hole to the Galactic Environment (Princeton, NJ: Princeton Univ. Press)

Liu Z., Yuan W., Lu Y., Carrera F.J., Falocco S., Dong X.-B., 2016, MNRAS, 463,684

Markoff S., Nowak M.A., 2004, ApJ, 609, 972

Morales Teixeira D., Fragile P.C., Zhuravlev V.V., Ivanov P.B., 2014, ApJ, 796, 103

Penna R.F., Sądowski A., Kulkarni A.K., Narayan R., 2013, MNRAS, 428, 2255

Reis R.C., Miller J.M., 2013, ApJ, 769, L7

Reynolds C.S., Fabian A.C., 2008, ApJ, 675, 1048

Shakura N.I., Sunyaev R.A., 1973, A\&A, 24, 337

Stella L., Rosner R., 1984, ApJ, 277, 312

Svennson R., Zdziarski A.A., 1994, ApJ, 436, 599

Svoboda J., Dovčiak M., Goosman R.W., Jethwa P., Karas V., Minuitti G., Guainazzi M., 2012, A\&A, 545, A106

Vasudevan R.V., Fabian A.C., 2007, MNRAS, 381, 1235

Vincent F.H., Różańska A., Zdziarski A.A., Madej J., 2016, A\&A, 590, A132

Wilkins D.R., Fabian A.C., 2012, MNRAS, 424, 1284

Wilkins D.R., Fabian A.C., 2015, MNRAS, 454, 4440

This paper has been typeset from a $\mathrm{T}_{\mathrm{E}} \mathrm{X} / \mathrm{LAT}_{\mathrm{E}} \mathrm{X}$ file prepared by the author. 